and being HSV2 seropositive at baseline (IRR1.7, 95\% CI 0.99 to 2.8, $\mathrm{p}=0.055$ ) were associated with an increased risk of seroconversion in younger women. In older women, decreasing partner contribution to the household economy (IRR 1.3, 95\% CI 1 to 1.7) and Trichomonas vaginalis infection at enrolment (IRR 2.3, 95\% CI 1.1 to 4.9) were associated with increased risk of HIV acquisition. Older women who reported condom use at last sex act had a greater risk of acquiring HIV (IRR 2.8, 95\% CI 1.3 to 5.9), which could reflect high risk sexual behaviour in this group. Reporting multiple partners was associated with increased risk of $\mathrm{HIV}$ acquisition in both younger (2.6, 1.3 to 5.3 ) and older women (2.8, 1.3 to 6.1$)$.

Conclusions STIs remain an important marker of ongoing HIV risk. Interventions that address the economic context of women's HIV risk will be important to evaluate. Additional investigation using multivariate analysis may elucidate these findings further.

\section{P1-S5.15 CONTRIBUTION OF CONDOM BREAKAGE TO THE HIV EPIDEMIC AMING MEN WHO HAVE SEX WITH MEN IN KARNATAKA, INDIA}

doi:10.1136/sextrans-2011-050108.193

${ }^{1} \mathrm{M}$ Pickles, ${ }^{2} \mathrm{P}$ Vickerman, ${ }^{3} \mathrm{~J}$ Bradley, ${ }^{4} \mathrm{~B} M$ Ramesh, ${ }^{4} \mathrm{~S}$ Isac, ${ }^{5} \mathrm{R}$ Washington, ${ }^{2} \mathrm{H}$ Prudden, ${ }^{2} \mathrm{~K}$ Mitchell, ${ }^{6} \mathrm{M}$ Alary, ${ }^{1} \mathrm{M} \mathrm{C}$ Boily. ${ }^{1}$ Imperial College, London, UK; ${ }^{2}$ LSHTM, UK; ${ }^{3}$ CHARME-India project, Bangalore, India; ${ }^{4}$ Karnataka Health Promotion Trust, Bangalore, India; ${ }^{5}$ St. John's Medical College Bangalore, India; ${ }^{6}$ Centre Hospiltalier Affilié Universitaire de Québec, Quebec, Canada

Background Condom promotion among female sex workers (FSWs) and men who have sex with men (MSM) is one of the key components of the Avahan HIV intervention currently underway in India. High rates of consistent condom use are reported across sites. However, condoms are only effective if they do not break or slip during intercourse, which occur frequently, especially in MSM.

Methods We use a deterministic compartmental mathematical model of MSM divided into high and low risk, with average condom use increasing over time, to investigate how breakage influences the course of the epidemic. High-risk MSM (HRMSM) sell sex or have many casual partners, and typically have mostly receptive anal sex. Prior parameter ranges (including condom use) for the model are based on one round of cross-sectional behavioural and biological (IBBA) surveys of HRMSM from four districts in Karnataka state in 2008. In these surveys $16.7 \%$ (82/491) of MSM report condom breakage in the last sex act. Condom use increased to $93 \%$ by 2008 , and was assumed to be constant afterwards. The prior ranges are sampled using Latin hypercube sampling. Model runs that agree with measured HRMSM HIV prevalence (12.7-18.9\%) provide the posterior parameter set fitting the data, and are used to make predictions of HIV prevalence with and without condom breakage after the start of the intervention in 2004.

Results Abstract P1-S5.15 figure 1 shows change in high/low risk prevalences with and without condom breakage. Even with high rates of condom breakage, HIV prevalence among MSM declines from $16.7 \%$ (95\% CI 12.7 to $20.8 \%$ ) in 2004 to $7.9 \%$ (5.9 to $9.8 \%$ ) in HRMSM by 2015. Without condom breakage HIV prevalence declines faster, to $5.6 \%$ (4.2 to $6.9 \%$ ) by 2015 . Compared to no condom breakage, there will be $10.3 \%(10.7 \%)$ more cumulative infections with breakage in high-risk (low-risk) MSM from 2004 until 2015. The annual extra fraction of new infections due to condom breakage increases from $4.1 \%$ (4.3\%), in 2004, to $158.9 \%$ (165.3\%), in 2015 in high-risk (low-risk) MSM, although the absolute number of infections due to condom breakage decreases as the epidemic is declining and condom use increases.

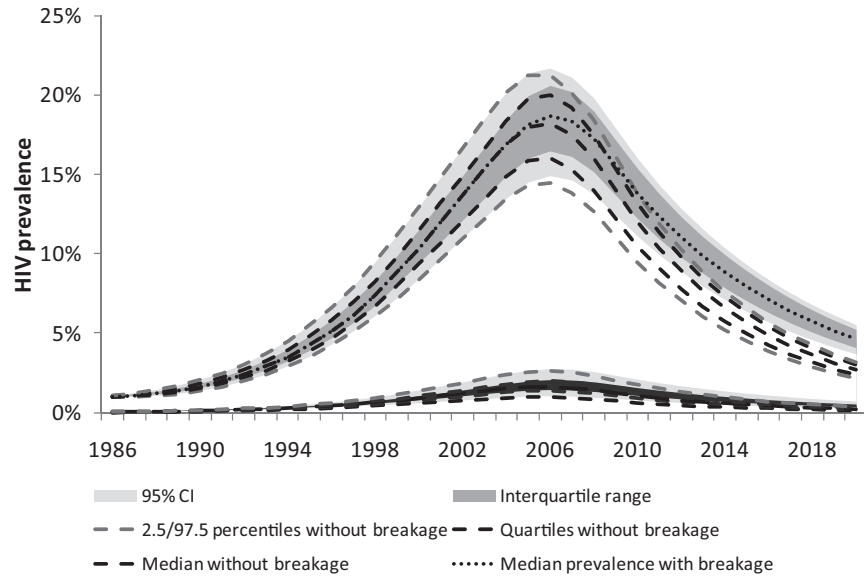

Abstract P1-S5.15 Figure 1 HIV prevalence against time for high-risk MSM (upper curves)and low-risk MSM (lower curves). Shaded region shows 2.5-97.5 percentiles and IQR with breakage. The dashed lines show the 2.5, 97.5 percentiles and quartiles without condom breakage.

Conclusions HIV prevalence is projected to decline in MSM in Karnataka, given high rates of reported condom use, even with frequent breakage. However, there will be a large fraction of extra infections due to condom breakage. HIV intervention programmes should examine reasons for high rates of breakage and take steps to address this.

\section{P1-S5.16 INVESTIGATING SELF-REPORTED LEVEL OF CONDOM USE AND CONDOM USE IN LAST ACT AMONG HIGH-RISK GROUPS IN SOUTHERN INDIA}

doi:10.1136/sextrans-2011-050108.194

${ }^{1} \mathrm{M}$ Pickles, ${ }^{2} \mathrm{R}$ Adhikary, ${ }^{3} \mathrm{M}$ Mainkar, ${ }^{3} \mathrm{R} S \mathrm{P},{ }^{4} \mathrm{~K}$ Deering, ${ }^{5} \mathrm{~S}$ Verma, ${ }^{1} \mathrm{M} \mathrm{C}$ Boily, ${ }^{6} \mathrm{P}$ Vickerman, ${ }^{7} \mathrm{M}$ Alary. ${ }^{1}$ Imperial College, London, UK; ${ }^{2}$ Family Health International, India; ${ }^{3}$ National AIDS Research Institute, Pune, India; ${ }^{4}$ University of British Columbia, Canada; ${ }^{5}$ Karnataka Health Promotion Trust, Bangalore, India; ${ }^{6}$ LSHTM, UK; ${ }^{7}$ Centre Hospiltalier Affilié Universitaire de Québec, Quebec, Canada

Background Measuring consistent condom use (CCU) is crucial in understanding the trajectory of the HIV epidemic and in evaluating HIV preventive interventions that promote condom use. We examine how one measure of CCU varies across different populations, and compare it to condom use in the last sex act.

Methods Using data from cross-sectional behavioural and biological surveys (2005-2007), we compare responses concerning selfreported level of condom use and condom use in the last act by female sex workers (FSWs), their clients, and men who have sex with men (MSM) in districts from Andhra Pradesh, Maharashtra and Tamil Nadu states in India. For FSWs/clients, the question related to sex with occasional clients/FSWs. For MSM, the question was for non-commercial, non-regular male partners. The time period was unspecified. Levels of condom use were defined as every time", "most of the time/sometimes", and "never". We define CCU as those who reply "every time".

Results $95.2 \%$ of all FSWs reported using a condom in the last act, compared to $72.9 \%$ of clients and $80.0 \%$ MSM. However, only $36.7 \%$ of clients and $29.6 \%$ of MSM reported CCU compared to $80.0 \%$ of FSWs. Abstract P1-S5-16 table 1 shows condom use in the last act stratified by reported level of condom use. For all populations reporting CCU "always", condom use in the last act was $>98 \%$. Of those who reported "most of the time/sometimes", there was a significant difference between condom use in the last act between clients and the other groups ( $\chi^{2}$ test, 